\title{
Multiple perforations associated with ulcerative colitis
}

\section{Introduction}

The term inflammatory bowel disease (IBD) includes two disorders of unknown etiology, ulcerative colitis (UC) and Crohn's disease (CD), which are defined according to clinical, radiological, endoscopic and histological criteria. ${ }^{1}$ The association between toxic colitis and perforation occurs in approximately $2 \%$ of patients with UC and is associated with considerable mortality rates, which can range from $27 \%$ to $57 \%$, which increase as the time between perforations is prolonged and the surgical intervention ${ }^{1}$. Surgical intervention in chronic ulcerative colitis is typically reserved for failure of medium therapy, exacerbation of the condition such as toxic colitis, perforation, bleeding, The development of structures or neoplasia. Level of Evidence III. Degree of recommendation A. ${ }^{1}$ Severe acute ulcerative colitis is one of the few emergencies in gastroenterology. The mortality rate has dropped from $70 \%$ in 1933 to $20-25 \%$ in the 50 's, when the importance of opportune emergency colectomy was recognized for the first time. ${ }^{2}$ Subsequently, the mortality rate was further reduced ${ }^{3}$ to $7 \%$ with the introduction of corticosteroids and eventually to $1 \%$ wax at present. ${ }^{4}$

Keywords: mortality rates, emergency colectomy, crohn's disease, perianal pain, burning, anorexia, asthenia, adynamia, weight loss, hemorrhoids, non-thrombosed, bleeding,

\section{Objective}

To document a clinical case presented in a patient of the General Hospital Dr. Dario Fernandez Fierro population as a case report as well as to discuss the management with respect to international literature.

\section{Case report}

This is a 51-year-old female patient, who began her condition 30 days ago with perianal pain, presence of burning-type stools without active bleeding at that time, reporting a self-exploration tumor in the right perianal region, reason for which arnica was washed. and water, secondary to which refers moderate bleeding with little suppuration; subsequently referred pain and bleeding with the evacuations, go to the emergency department, medical management is indicated based on topic not specified with partial improvement of pain, persisting bleeding, anorexia, asthenia, adynamia and weight loss without referring precise figure. Physical examination, soft abdomen, depressable, painful on palpation in hypogastrium, without palpable masses, without data of peritoneal irritation or plastrones. Peristalsis present, normoactive. Perianal region reporting a closed anus with abscess drained in left anterolateral region without bleeding, or suppuration with grade 2 hemorrhoids, non-thrombosed, painless. Rectal examination revealed a thickened posterior hemorrhoidal package without active bleeding. Laboratory tests with lengthening of coagulation times with TP: INR 25.9: 1.89 TTP: 39.5, blood count with leukocytosis, neutrophilia, decrease in hemoglobin and decrease in hematocrit LEU: 21.5 NEU: 97.6 LIN 2.3 HB: 8.6 HTO: 25.9 PLQ :
Volume 2 Issue 4 - 2018

\author{
Tolentino Gonzalez Christian Stefan,' \\ Ramirez Garcia Maria de Lourdes, ${ }^{2}$ De \\ la Rosa Jimenez Antonio, ${ }^{3}$ Carrillo Rivera \\ Jorge, ${ }^{4}$ Luis Antonio Reyes Quijano' \\ 'General Surgeon, Hospital ISSSTE, Mexico \\ 2Medical Doctor, Private Practice, Mexico \\ ${ }^{3}$ Coloproctologist, Hospital General, Mexico \\ ${ }^{4}$ Oral and Maxilofacial Surgeon, Hospital General, Mexico
}

Correspondence: Christian Stefan Tolentino Gonzalez, General Surgeon, Hospital ISSSTE, San Francisco 524, Col. Del Valle C.P. 03100 Ciudad de México, Tel55239149, Email dr.ctolentino@hotmail.com

Received: June 28, 2018 | Published: June 28, 2018

290 TAC simple and contrasted abdominopelvica and abdominal free LAPE + SUBTOTAL COLECTOMY + ILEOSTOMY TERMINAL + RIGHT OOFORECTOMY + HARTMAN PROCEDURE is performed (Figure 1), by the diagnosis of PERFORATIONS IN COLON the transoperative findings are: "Perforations in caecum 1 and $1.5 \mathrm{~cm}$, ascending colon of approx. $2 \mathrm{~cm}$, sigmoid of approx. 1.5 $\mathrm{cm}$ (Figure 2), emplastronadas with annexes, right annex with changes of coloration, liquid of intestinal characteristics, approx. 300 cc. " Histopathological report: Macroscopic description, appendix, caecum and colon with loss of continuity solution located $8 \mathrm{~cm}$ ileocecal valve and a second $15 \mathrm{~cm}$, serous, depulidated, hemorrhagic granular surface with purulent cream, ascending colon with fibro-fatty adhesion, localized occlusion zone to $23 \mathrm{~cm}$. Anatomopathological diagnosis, nonspecific chronic ulcerative colitis in acute and perforated phase, transmural hemorrhagic infarction, acute and chronic non-specific peritonitis, acute periappendicitis.

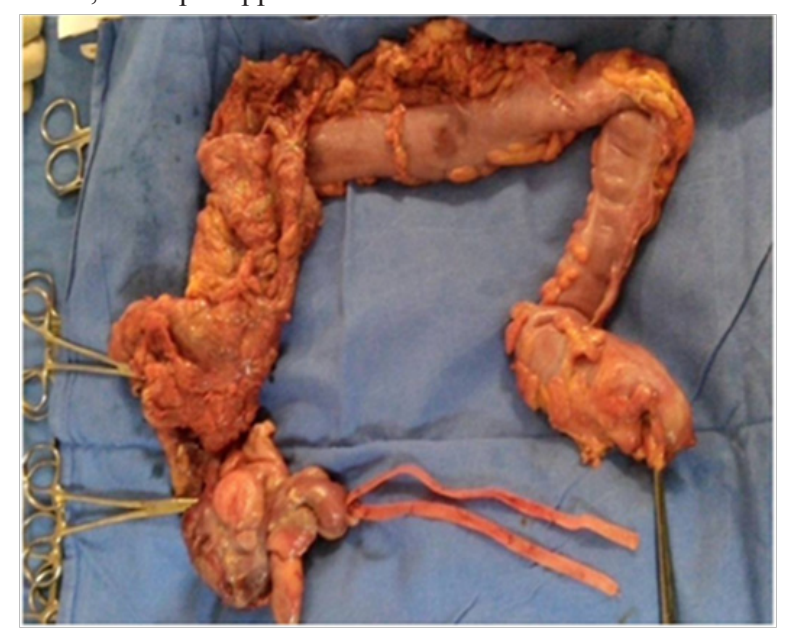

Figure I Air is reported, free fluid in the pelvic cavity, no tumor is observed in the pelvic cavity. 


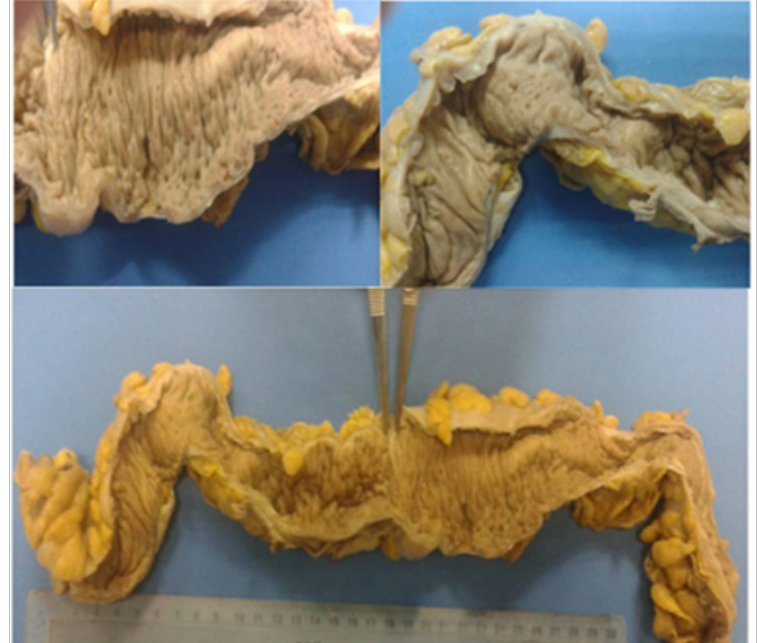

Figure 2 Histopathological report: Macroscopic description, appendix, caecum and colon with loss of continuity solution located $8 \mathrm{~cm}$ ileocecal valve and a second $15 \mathrm{~cm}$, serous, depulidated, hemorrhagic granular surface with purulent cream, ascending colon with fibro-fatty adhesion, localized occlusion zone to $23 \mathrm{~cm}$.

\section{Discussion}

Several potentially surgical complications in UC are described, among which the main ones are fulminating colitis, toxic megacolon, perforation and bleeding. ${ }^{1}$ It is important to know how to identify them to guide an appropriate treatment. In the case of this article, intestinal perforation represents a surgical emergency and entails a considerable morbidity and fortunately, contrary to what previously happened, a decreasing mortality rate. ${ }^{5}$ The intestinal perforation associated with non-specific chronic ulcerative colitis, without the presence of toxic megacolon, is not reported in the literature. In the case we present, the patient debuts with multiple intestinal perforations (caecum, ascending, sigmoid), which is an indication in any patient for emergency surgical treatment. Currently the initial treatment in UC is medical treatment (parenteral solutions, antibiotics and intravenous corticosteroids), surgical treatment is left to patients who have a refractory response to medical treatment or in patients who require urgent surgical treatment, as in this case. ${ }^{2}$

The perforation represents the most serious complication in UC and is usually associated with instrumentation (colonoscopy) or with the presence of toxic megacolon and delay in diagnosis or attention, due to the consequent presence of perforation. ${ }^{6}$ The association between toxic colitis and perforation occurs in approximately $2 \%$ of patients with UC and is associated with considerable mortality rates, which can range from $27 \%$ to $57 \%,{ }^{7}$ which increase as the time between perforation is prolonged and the surgical intervention. ${ }^{4}$

\section{Conclusion}

The absolute indications for surgery are massive hemorrhage, perforation and documented evidence or suspicion of colon carcinoma. (Level of Evidence III, Degree of recommendation A). ${ }^{1}$ Therefore, in light of these findings, treatment should not be delayed urgently. Another indication for surgery is severe colitis with or without toxic megacolon that does not respond to conventional treatment and in those patients with a less serious but medically intractable disease or due to the presence of serious side effects of medical management. (Level of Evidence III, Degree of recommendation A). ${ }^{1}$ In this case, patients who present a poor response to medical treatment should be identified and appropriately registered to prevent, through elective surgery, the possible complications that may occur, such as those already described.

\section{Acknowledgements}

None.

\section{Conflict of interest}

The author declares there is no conflict of interest.

\section{References}

1. Yamamoto-Furusho JK, Bosques-PadillaF, Galiano MT, et al. Diagnóstico y tratamiento de la enfermedad inflamatoria intestinal: Primer Consenso Latinoamericano de la Pan American Crohn's and Colitis Organisation. Revista de Gastroenterología de México. 2017;82(1):46-84.

2. Yu YR, Rodriguez JR. Clinical presentation of Crohn's, ulcerative colitis, andindeterminate colitis: symptoms, extraintestinal manifestations, and diseasephenotypes. Semin Pediatr Surg. 2017;26(6):349-355.

3. Soon IS, Molodecky NA, Rabi DM, et al. The relationship between urban environment and the inflammatory bowel diseases: a systematic review and meta-analysis. BMC Gastroenterology. 2012;12:51.

4. Douglas Overbey, Henry Govekar, Csaba Gajdos. Surgical management of colonic perforation due to ulcerative colitis during pregnancy: Report of a case. World J Gastrointest Surg. 2014;6(10): 201-203.

5. Masaki T, Kishiki T, Kojima K, et al. Recent trends (2016-2017) in the treatment of inflammatory bowel disease. Ann Gastroenterol Surg. 2018;2(4):282-288.

6. Uchino M, Ikeuchi H, Sugita A, et al. Pouch functional outcomes after restorative proctocolectomy with ileal-pouch reconstruction in patients with ulcerative colitis: Japanese multi-center nationwide cohort study. $J$ Gastroenterol. 2017;53(5):642-51.

7. Team E. The Invisible Journal of IBD: 2017 in America Results. 2018. 